\title{
Potentiation of attentional enhancement in the pigeon produced by a "blocking" stimulus
}

\author{
LEHA ROBLES, ROBERT J. NEWLIN, and DAVID R. THOMAS \\ University of Colorado, Boulder, Colorado 80309
}

\begin{abstract}
In Stage 1, four groups of pigeons were given true discrimination (TD) and four groups were given pseudodiscrimination (PD) training along one of two dimensions orthogonal to wavelength. In Stage 2, all groups received single stimulus (SS) training with a wavelength cue compounded with the former S+ for four of the groups (two TD and Two PD) and with a novel stimulus for the other four (two TD and two PD). For all groups, the SS training was followed by a wavelength generalization test in order to assess the control acquired by the (incidental) wavelength cue during Stage 2. The presence of the former S+ should have blocked the acquisition of control by the wavelength cue leading to flat generalization gradients in the appropriate TD groups. To the contrary, however, these groups showed the sharpest wavelength gradients, i.e., rather than blocking, potentiation was found. One possible interpretation is that the superimposition of the previous $\mathrm{S}+$ served as a reminder of previous TD training, thereby enhancing the transfer of an attentive set from Stage 1 to Stage 2. An alternative conditioning interpretation, suggested in a 1975 paper by Mackintosh, can also be extended to encompass these results.
\end{abstract}

Many studies (Bresnahan, 1970; Hall \& Honig, 1974; Honig, 1969; Mackintosh \& Honig, 1970; Thomas, Freeman, Svinicki, Burr, \& Lyons, 1970; Thomas, Burr, \& Eck, 1970; Turner \& Mackintosh, 1972) have demonstrated that free operant discrimination training (called TD for true discrimination) along one dimension sharpens generalization gradients obtained along a second, orthogonal stimulus dimension, relative to either a single stimulus (SS) control group or one given nondifferential training (called PD for pseudodiscrimination). To take a particular example, in Thomas et al. (1970, Experiment 2) pigeons were given either TD or PD training in Stage 1 with vertical and oblique lines, followed by SS training in Stage 2 with a green stimulus on which the previously positive (for TD subjects) vertical line was superimposed. All subjects then received a wavelength generalization test in extinction, with the TD group yielding the sharper gradient. This result, called the TD enhancement effect, has been interpreted by Thomas et al. (1970) as evidence for a process of general attention, that is, in discrimination training TD subjects presumably learn about the importance of stimuli in general, whereas in nondifferential training PD subjects learn about the insignificance of stimuli. This set or expectancy is applied to stimuli present during the discrimination

This research was supported by NIH Grant HD-03486 and NSF Grant BNS 78-01407 to David R. Thomas. It was presented at the April 1978 meeting of the Rocky Mountain Psychological Association in Denver. The authors are indebted to K. Geoffrey White and Thomas Moye for many helpful suggestions that were incorporated in the manuscript. or nondifferential training as well as to those experienced later, as in the reference experiment described above.

Mackintosh (1977) has proposed an alternative interpretation of the TD enhancement effect based on the principles of blocking and masking. This explanation assumes that only the process of competition between stimuli with respect to control over behavior is necessary to integrate the TD enhancement effect with other findings in the area of stimulus control. In the Thomas et al. (1970) experiment, the presence of the previous $\mathrm{S}+$ in Stage 2 should block acquisition of control by the green stimulus in the TD group in comparison to the PD group for which the vertical line was a less valid stimulus. Since the results of the generalization test suggest more control by green in the TD than in the PD group, some other, unspecified cues must be controlling behavior. These cues are all the stimuli common to both stages of the experiment, called the "context." During TD training in Stage 1, control by these stimuli is suppressed (or overshadowed) by the presence of the valid S + and S - stimuli. During PD training, when there are no valid stimuli, all stimuli, including the context, come to control behavior. During generalization testing, the control over behavior by these contextual stimuli for PD subjects masks the greater control acquired by the green stimulus.

There is evidence in the literature for processes of blocking (cf. Mackintosh \& Honig, 1970) and masking (Farthing, 1972; Thomas, Svinicki, \& Svinicki, 1970) affecting the slope of generalization 
gradients. The reality of these effects is not in question here. However, the claim that the masking effect is so great as to completely obliterate the blocking effect in studies such as the reference experiment of Thomas et al. (1970) is entirely post hoc.

Since TD and PD groups presumably differ in both blocking and masking, the normal experimental design, which compares gradients following TD and PD training, does not permit the separate analysis of these processes. Blocking can be studied independently from masking, however, by comparing only TD groups, which are similar from the standpoint that control by context should be minimal and thus masking should be minimal in each. Where TD groups differ in the presence vs. absence of a potential blocking stimulus, differential blocking can reasonably be expected, and, in the absence of masking, such differences should be reflected in variations in gradient slope.

Consider the following pilot experiment. Two groups of pigeons $(n=8)$ were given TD training. For Group 1, S + was a vertical white line on a dark surround; $\mathrm{S}-$ was a horizontal line. For Group 2, $\mathrm{S}+$ and $\mathrm{S}-$ were a brightly or a dimly illuminated key, respectively. Stage 2 , which was the same for both groups, involved SS training with a white vertical line (the former $S+$ for Group 1) superimposed on a blue-green surround. Wavelength generalization testing followed in extinction.

The presence of the former $\mathrm{S}+$ should have blocked acquisition of control by the incidental blue-green stimulus in Group 1 relative to Group 2, in which the vertical line was simply a novel stimulus presumably orthogonal to the previous $\mathrm{S}+$. Since masking by the context should have been minimal and comparable for both groups, blocking should have been revealed by a flatter wavelength gradient in Group 1. If, as suggested by Mackintosh (1977), masking were the overriding factor determining gradient slope, the two gradients might have been comparable in slope. To the contrary, however, Group 1 showed a substantially sharper gradient, indicating that the presence of the previous $\mathrm{S}+$ potentiated the acquisition of control by wavelength rather than blocked it. This suggests that: (1) blocking may not occur in this paradigm, and/or (2) there is a more potent process which obscures any possible finding of blocking and results in the sharper gradient in Group 1.

Before developing alternative interpretations of the pilot experiment, it seemed appropriate to replicate and extend its findings. In the present paper, we report an extensive replication which accomplishes two additional objectives. First, it counterbalances such that each $\mathrm{S}+$ is also a novel stimulus in Stage 2 for a different group. Second, it includes PD groups, thereby enabling us to ask whether the size of the TD enhancement effect, relative to an appropriate control, is also increased by maintaining the former $\mathrm{S}+$ during Stage 2 .

\section{METHOD}

\section{Subjects}

The subjects were 84 naive pigeons obtained from a local supplier and maintained at $70 \%-75 \%$ of their ad-lib weights during the experiment. The subjects were housed in individual cages where grit and water were continuously available. They were maintained on a 16 -h-light/8-h-dark cycle, sessions occurring between 2 and $8 \mathrm{~h}$ after lights on.

\section{Apparatus}

Four similar sound-attenuating ice chests, with interior dimensions of $31 \times 35 \times 36 \mathrm{~cm}$, served as experimental chambers. Each chamber had a wire mesh floor, and one wall, constructed of aluminum with a 2.2 -cm-diam translucent plastic response key centered $20 \mathrm{~cm}$ above the floor, constituted an "intelligence panel." Approximately $13 \mathrm{~cm}$ directly below the key, a $4.5 \times$ $6 \mathrm{~cm}$ aperture allowed access to mixed grain from a solenoidoperated hopper. Behind the front wall directly to the right of the aperture was a 10 -cm-diam speaker through which white noise was delivered. A fan, mounted behind the front wall, provided ventilation as well as additional masking noise. Noise level in the chamber was measured at $80 \mathrm{~dB}$ (SPL).

Stimuli were projected onto a translucent screen on the front of IEE (Series 0010) in-line display cells mounted $.635 \mathrm{~cm}$ behind the key aperture. Display cells fitted with Kodak Wratten filters Nos. $75,74,99$, and 73 provided chromatic stimuli with nominal peak wavelength transmission of $490,538,555$, and $576 \mathrm{~nm}$, respectively. The display cell allowed projection of a .2 -cm-wide white line on a black surround with angular orientations of $90 \mathrm{deg}$ (vertical) and 0 deg (horizontal). The display cells could also present either the top or the bottom half of the key dark and the other half lighted white. Both the line and half-light/ half-dark stimuli could be combined with the chromatic stimuli so that the previously dark areas were illuminated with the chromatic stimuli. Rear projection of these stimuli was accomplished by illuminating miniature 14-V lamps (No. 1815). Chamber illumination was provided by lighting a single No. 1829 lamp located behind a $3 \times 31 \mathrm{~cm}$ strip of diffusing Plexiglas which extended across the chamber above the intelligence panel. The only other source of illumination in the chambers was provided by an ESB 24-V lamp that was above the food hopper and was illuminated during the hopper presentation.

\section{Procedure}

On the 1st day of preliminary training, all birds were magazinetrained with half of the birds hand-shaped to peck the vertical line and the other half shaped in the presence of a top-halflight/bottom-half-dark cue. All birds were allowed to earn 30 reinforcers delivered on a continuous reinforcement schedule. The next 50 reinforcers were delivered on a reinforcement schedule that increased from continuous to an average interreinforcement interval of $30 \mathrm{sec}$. On the 2 nd day, the birds were allowed to earn 10 reinforcers delivered on a continuous reinforcement schedule and 40 more reinforcers on a schedule whose average interreinforcement interval increased to $30 \mathrm{sec}$. On the $3 \mathrm{rd}$ day, all birds earned 30 total reinforcers delivered while a variableinterval 30-sec-schedule was in effect. On the 4th day, all birds received a single 20 -min session in which the shaping stimulus was presented for 90 -sec periods separated by $10-\mathrm{sec}$ blackouts and responses were reinforced with $3-\mathrm{sec}$ access to grain on a constant probability 1-min schedule (Catania \& Reynolds, 1968) in which interreinforcement intervals ranged from 3 to $160 \mathrm{sec}$. 
Subsequent assignment of subjects to eight groups $(\mathrm{n}=10$ for half and $n=11$ for the other half) was accomplished by matching their response rates during the fourth session.

The complete design of the experiment is illustrated in Figure 1.

Stage 1. In Stage 1, four groups of birds were trained with the line-angle stimuli and four groups were trained with the half-light/ half-dark stimuli. Within each training stimulus condition, half of the subjects received TD training and half received PD training. In the TD training, the vertical line or the top-half-light stimulus $(\mathrm{S}+)$ and the horizontal line or the top-half-dark stimulus (S-) were presented for 90 -sec periods separated by 10 blackouts. During $\mathrm{S}+$ periods, responding was reinforced on a constant probability $1-$ min schedule and responding during $S-$ periods was not reinforced. Twelve $S+$ and $S-$ periods were presented daily for a minimum of 4 days and until a performance criterion of more than $90 \%$ of the total responses in $\mathrm{S}+$ periods was met. PD training was programmed to present the same reinforcement patterning as the discrimination training. The training stimuli were both paired with reinforcement (a pseudorandom) $50 \%$ of the time and with nonreinforcement the other $50 \%$ of the time.

Stage 2. Each PD bird was paired with a TD bird and began Stage 2 training when the TD bird had reached the performance criterion of Stage 1 training. In Stage 2, all groups received four sessions of single-stimulus training. For half of the groups (two TD, two PD), the vertical line was presented on a $490-\mathrm{nm}$ background for 1290 -sec stimulus periods separated by 10 -sec blackouts, while for the other four groups (two TD, two PD), the top-half-light stimulus was compounded with the $490-\mathrm{nm}$ stimulus, that is, the top half was white and the bottom half blue-green. Responding was reinforced on a constant probability 1-min schedule. For two pairs of matched TD and PD groups (one pair pretrained with lines and one pair pretrained with the half-light/half-dark cues), a former $\mathrm{S}+$ stimulus from Stage 1 was present throughout Stage 2 training (Groups $S+T D$ and $S+P D)$. The other two pairs of matched TD and PD groups

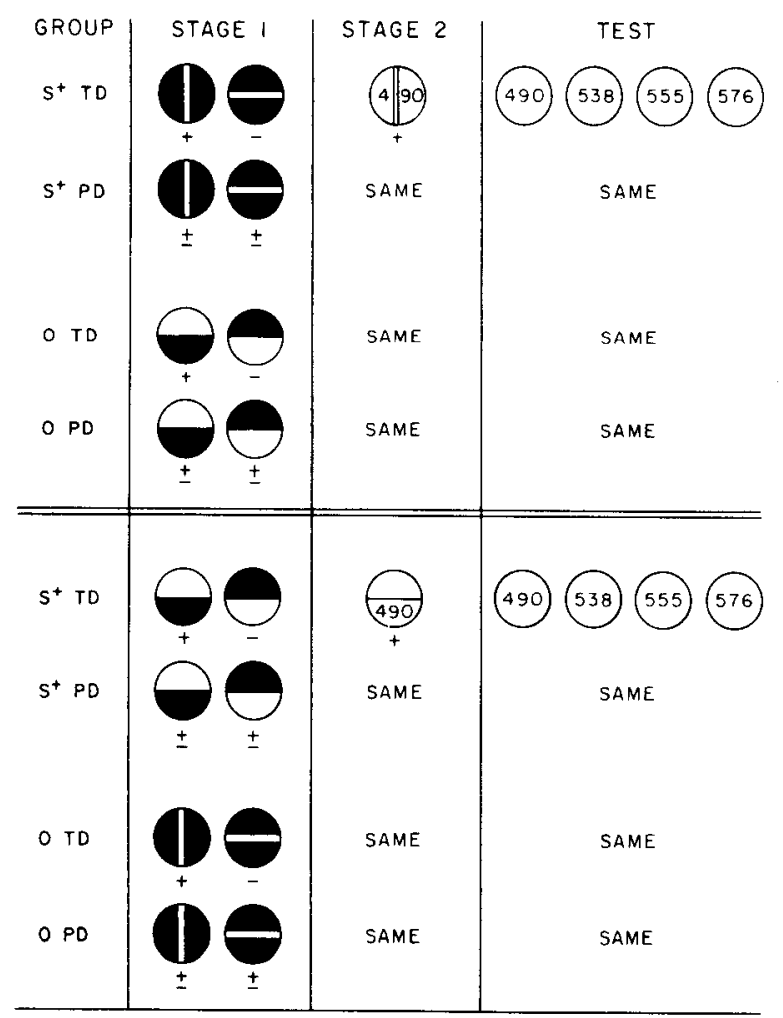

Figure 1. A schematic representation of the procedure. (one pair pretrained with lines and one pair pretrained with half-light/half-dark cues) experienced the tested dimension in the presence of a novel cue, presumably orthogonal to the previous $\mathrm{S}+$ (Groups OTD and OPD).

Stage 3. Stage 3 consisted of 1 day of generalization testing in extinction for all groups. Four wavelengths, 490, 538, 555, and $576 \mathrm{~nm}$, were presented once in each of 12 randomized blocks. The stimuli lasted $30 \mathrm{sec}$ each and were separated by $10-\mathrm{sec}$ blackouts. No other stimuli were projected on the display $\mathrm{key}$, and there was no warm-up period before the test.

\section{RESULTS}

\section{Stage 1}

The TD groups' terminal discrimination ratios based on the mean percent of total responses made to the $\mathrm{S}+$ stimulus on the last day of TD training ranged from 96.4 (S+ TD with lines) to 98.5 (S+ TD with half-light/half-dark cues) and did not differ between groups $(F<1)$. A mean of 4.2 sessions was required to reach criterion (range 4 to 6 sessions for individual subjects), and mean responses to $\mathrm{S}+$ on the last day of TD training ranged from 1,240 (OTD with half-light/half-dark cues) to 1,601 (OTD with lines). The PD groups did not differ $(F<1)$ in final Stage 1 discrimination ratios based on the mean percent of total responses made to the matched TD subject's $\mathrm{S}+$ stimulus. Discrimination ratios ranged from a mean of 52.2 (OPD with lines) to 58.4 (OPD with half-light/half-dark cues) with 31 of 38 subjects having ratios above $50 \%$, indicating a slight tendency to prefer the stimulus to which they were shaped $(p<.01)$. Mean responses to the matched TD subject's $S+$ ranged from 760 (OPD with lines) to 1,018 ( $\mathrm{S}+\mathrm{PD}$ with lines).

\section{Stage 2}

Response rates across all groups at the end of Stage 2 training were approximately equal $(F<1)$ and ranged from a mean of 946 responses (OPD pretrained with half-light/half-dark cues) to a mean of 1,312 responses (OTD pretrained with lines).

\section{Stage 3}

Subjects that respond erratically during testing tend to give irregular and unreliable gradients. For this reason, subjects that did not respond on at least half of the 12 blocks of test stimuli were dropped from the experiment, along with their matched controls. Two OTD subjects pretrained with lines and one OTD and one OPD subject pretrained with half-light/half-dark cues failed to reach the criterion of responding on half of the test blocks and were dropped from the experiment along with their matched subjects. The data for these subjects has not been included in any of the analyses.

The wavelength generalization gradients obtained in Stage 3 are presented graphically in Figures 2 and 3 . Figure 2 presents gradients following chro- 

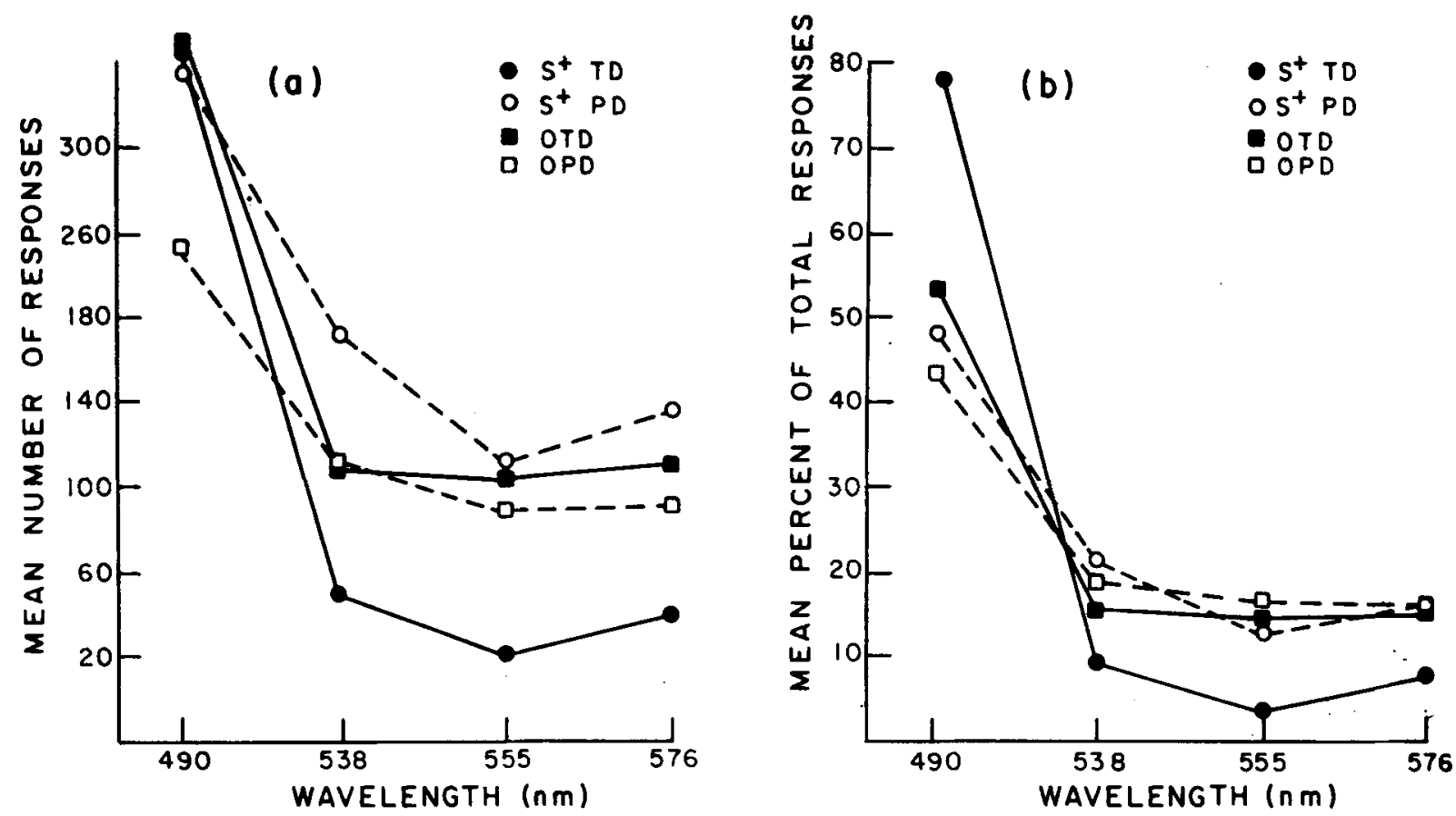

Figure 2. Absolute generalization gradients (Panel a) and relative generalization gradients (Panel b) of groups for which the vertical line was paired with the chromatic cue in Stage 2.
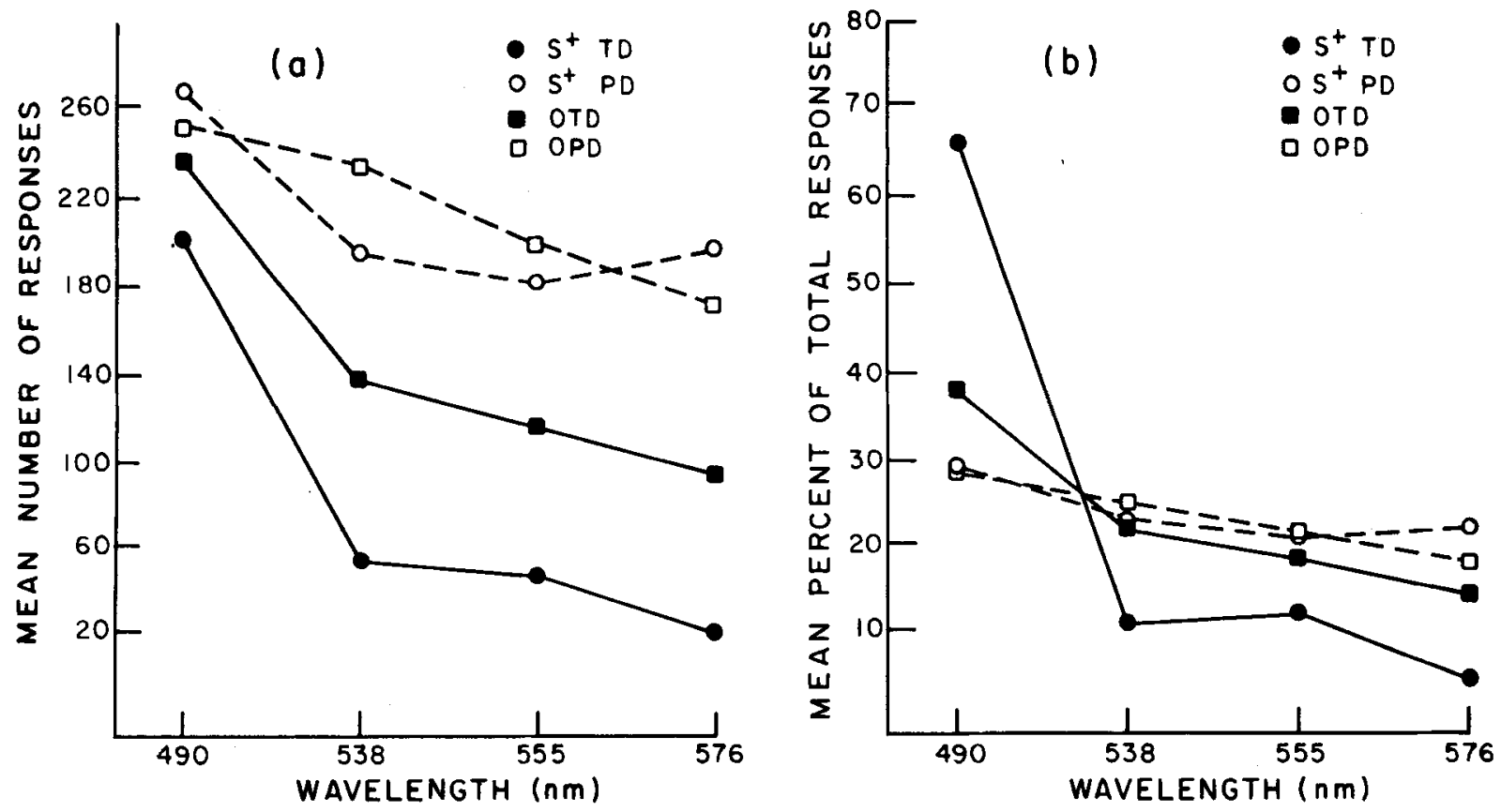

Figure 3. Absolute generalization gradients (Panel a) and relative generalization gradients (Panel b) of groups for which the top-half-light stimulus was paired with the chromatic cue in Stage 2.

matic cue learning in the presence of a vertical line, and Figure 3 presents gradients following chromatic cue learning with the top-half-light stimulus. Relative gradients based on the percentage of responses to each test value are depicted in Figures $2 b$ and $3 b$, while absolute gradients based on total responses to each test stimulus are presented in Figures 2a and 3a. Relative gradients constitute the primary measure of gradient slope, since absolute differences in level of responding in free operant situations can be so extreme as to result in group mean absolute gradients which are not representative of individual 
performance (cf. Thomas et al., 1970). With the transformation to relative gradients, each subject contributes equally to the shape of the group average gradient. Absolute gradients are also presented for comparison purposes.

It is clear in both Figures 2 and 3 that TD groups produced sharper gradients than PD groups. Also, TD groups for which the former $\mathrm{S}+$ was maintained during Stage 2 produced sharper gradients than the TD groups for which a novel stimulus was present in Stage 2. Statistical analysis was accomplished by analysis of variance in which Test Stimuli (four wavelengths), Pretraining (TD vs. PD), S+ (presence vs. absence), and Stimulus Dimension (vertical line or top-half-light cue paired with the chromatic cue in Stage 2) were the factors. Adjustments for unequal $\mathrm{n}$ were made according to the method of Edwards (1972). The relative measure analysis (Figures $2 \mathrm{~b}$ and $3 \mathrm{~b}$ ) revealed a reliable Test Stimulus by Pretraining interaction $[F(3,204)=26.28$, $\mathrm{p}<.01]$, which confirms that the TD gradients were sharper than the PD gradients. More importantly, a reliable Test Stimulus by $\mathrm{S}+$ interaction $[F(3,204)=12.86, p<.01]$ confirms that maintaining the former $\mathrm{S}+$ on the key during SS training results in enhanced control by the wavelength dimension. The three-way interaction between Test Stimulus, Pretraining, and $S+[F(3,204)=10.38$, $\mathrm{p}<.01]$ was also reliable, indicating that the effect of maintaining the $\mathrm{S}+$ was confined to the TD groups.

The analysis also revealed that the gradients of subjects for which the vertical line was paired with the chromatic cue in Stage 2 were sharper than those of the subjects for which the top-half-light cue was used $[F(3,204)=14.29, p<.01]$.

Analysis of the absolute gradients indicated further that PD subjects responded more during the test than did TD subjects $[F(1,68)=10.37, p<.01]$ and that $S+$ TD subjects responded least, that is, the Pretraining by $S+$ interaction was significant $[F(1,68)$ $=5.25, \mathrm{p}<.05]$.

Finally, a significant effect for Stimulus Dimension $[F(1,68)=4.03, p<.05]$ indicates that subjects for which the vertical line was paired with the chromatic cue in Stage 2 responded more during the test than did the subjects for which the top-half-light cue was used in Stage 2.

Although there were differences in level of responding and in the sharpness of gradients between stimulus dimensions, the important fact for present purposes is that with both stimulus dimensions the TD enhancement effect is larger following added cue training in the presence of the previous $\mathrm{S}+$.

\section{DISCUSSION}

The purpose of this study was to investigate blocking by a former $\mathrm{S}+$ where masking by context was equal and minimal according to a proposal by Mackintosh (1977). In contrast to the prediction of blocking, a potentiation of the TD enhancement effect was found when the former $\mathrm{S}+$ was maintained during SS training. These results must lead to a rejection of Mackintosh's (1977) proposal.

Our assumption that masking would be equal and minimal in the TD groups was based on Mackintosh's explicit statement to that effect, that is, "the incidental stimuli suppressed during the course of discrimination training remain suppressed when the original discriminative stimuli are removed" (1977, p. 498). In a further elaboration of this theory, Mackintosh (1975) did not make the assumption that incidental stimuli remain suppressed, but assumed that they slowly acquire associative strength during subsequent training. More specifically, if we assume that during the 4 days of SS training control by context increased in the orthogonal TD groups but did not reach the level of control in the PD groups, and we further assume that the presence of the vertical line blocked acquisition of control by both wavelength and contextual stimuli in the $\mathrm{S}+\mathrm{TD}$ groups, then these results could still be interpreted as differential masking by context during the test.

While available evidence does not unequivocally contradict a conditioning account of these experimental results, we believe that the evidence favoring such an account is tenuous. For example, blocking of the acquisition of stimulus control in an operant setting has only been demonstrated when the discriminative stimuli from Stage 1 are compounded with (and thus render redundant) the discriminative cues introduced in Stage 2 (cf. Mackintosh \& Honig, 1970). As far as we know, there has never been a demonstration of blocking taking place in subsequent SS training, yet that is what purportedly happens in Stage 2 in the present study. Secondly, masking of the expression of stimulus control has generally been a subtle effect requiring large groups or within-subject designs to achieve statistical significance. In the four published studies of which we are aware (Farthing, 1972; Newman \& Benefield, 1968; Thomas, Ernst, \& Andry, 1971; Thomas, Svinicki, \& Svinicki, 1970), a highly salient on-key stimulus, background color, masked control by a less salient dimension, line angle. There is no published report of control by key color being masked by any other stimulus, on-key or off.

The conditioning interpretation of the present experiment is decidedly unparsimonious, and postulates a complex interaction between two processes, blocking and masking, for which there is little independent evidence. Furthermore, its acceptance would require a complete revision in our conception of what the slope of a generalization gradient means. No longer does slope reflect the degree of control over behavior acquired by the 
tested dimension; instead, it reflects the extent of control exercised by unspecified (and perhaps unspecifiable) other stimuli in the test situation.

The results are not inconsistent with an extension of the general attention position of Thomas et al. (1970). As a set to attend to stimuli and be more responsive to stimulus change is transferred to a new situation, we should expect to find a generalization decrement in that set whenever transfer conditions are different from training conditions. When the former $\mathrm{S}+$ is maintained on the key, the situation is clearly more like previous training than when a novel stimulus is present on the key. Put another way, the former $\mathrm{S}+$ should serve as a powerful reminder of the previous training and thus enhance the set to attend. One problem with this analysis is that while there is a large effect in the TD case, one might also expect that a stimulus associated with PD training would remind the subject of a set to respond nondiscriminatively, thus yielding a flatter gradient for $\mathrm{S}+\mathrm{PD}$ subjects than for OPD. No such effect is evident in the data. A highly likely reason for this is that the PD flattening effect is generally smaller in magnitude and less stable than the TD enhancement effect, relative to a single stimulus (SS) control group, and therefore the brief training given may not have been sufficient to establish a nondiscriminative set. The uniformly slightly positive discrimination ratios in the PD groups suggest that those birds had not yet learned to treat their two stimuli equally. Thus, our PD groups are probably comparable to SS groups and there is no reason to believe that a reminder of prior SS training would have any effect on control acquired by an incidental stimulus during further SS training.

Whatever the ultimate explanation of this study may be, we have uncovered a previously unreported, robust, and perhaps counterintuitive phenomenon, the potentiation of the TD enhancement effect by a stimulus predicted to produce blocking. Further research will be needed to pursue the various implications of both the memory and the conditioning interpretations of this phenomenon.

\section{REFERENCES}

Breshahan, E. L. Effects of extradimensional pseudodiscrimination and discrimination training upon stimulus control. Journal of Experimental Psychology, 1970, 85, 155-156.

Catania, A. C., \& Reynolds, G. S. A quantitative analysis of the responding maintained by interval schedules of reinforcement. Journal of the Experimental Analysis of Behavior, 1968, $11,327-383$.

Edwards, A. L. Experimental design in psychological research. New York: Holt, Rinehart \& Winston, 1972.

Farthing, G. W. Overshadowing in the discrimination of successive compound stimuli. Psychonomic Science, 1972, 28, 29-32.

HALl, G., \& Honig, W. K. Stimulus control after extradimensional training in pigeons: A comparison of response contingent and noncontingent training procedures. Journal of Comparative and Physiological Psychology, 1974, 87, 945-952.

Honig, W. K. Attentional factors governing the slope of the generalization gradient. In R. M. Gilbert \& N. S. Sutherland (Eds.), Animal discrimination learning. London: Academic Press, 1969.

Mackintosh, N. J. A theory of attention. Psychological Review, 1975, 82, 276-298.

Mackintosh, N. J. Stimulus control: Attentional factors. In W. K. Honig \& J. E. R. Staddon (Eds.), Handbook of operant behavior. Englewood Cliffs, N.J: Prentice-Hall, 1977.

Mackintosh, N. J., \& Honig, W. K. Blocking and attentional enhancement in pigeons. Journal of Comparative and Physiological Psychology, 1970, 73, 78-85.

Newman, F. L., \& Benefield, R. L. Stimulus control, cue utilization, and attention: Effects of discrimination training. Journal of Comparative and Physiological Psychology, 1968, 66, 101-104.

Thomas, D. R., Burr, D. E. S., \& Eck, K. O. Stimulus selection in animal discrimination learning: An alternative interpretation. Journal of Experimental Psychology, 1970, 86, 53-62.

Thomas, D. R., ERNST, A. J., \& ANDRY, D. K. More on masking of stimulus control during generalization testing. Psychonomic Science, 1971, 23, 85-86.

Thomas, D. R., Freeman, F., Svinicki, J. G., Burr, D. E. S., \& Lyons, J. The effects of extradimensional training on stimulus generalization Journal of Experimental Psychology, 1970, 83, Whole No. 1, Pt. 2, 1-21.

Thomas, D. R., Svinicki, M. D., \& Svinicki, J. G. Masking of stimulus control during generalization testing. Journal of Experimental Psychology, 1970, 84, 479-482.

Turner, C., \& Mackintosh, N. J. Stimulus selection and irrelevant stimuli in discrimination learning in pigeons. Journal of Comparative and Physiological Psychology, 1972, 78, 1-19.

(Received for publication February 27, 1979; revision accepted August 31, 1979.) 